\title{
Non contact inspection of the fatigue damage state of carbon fiber reinforced polymer by optical surface roughness measurements
}

\author{
Pablo Zuluaga-Ramírez ${ }^{\mathrm{a}, \mathrm{b}, *}$, Malte Frövel ${ }^{\mathrm{a}}$, Tomás Belenguer ${ }^{\mathrm{a}}$, Félix Salazar ${ }^{\mathrm{b}}$ \\ ${ }^{a}$ Instituto Nacional de Técnica Aeroespacial, INTA, 28850, Torrejón de Ardoz, Madrid, Spain \\ ${ }^{\mathrm{b}}$ Department of Applied Physics (FARN), ETSI Minas, Universidad Politécnica de Madrid, 28003 Madrid, Spain
}

\begin{abstract}
A B S T R A C T
This work presents the evaluation of a new non-contact technique to assess the fatigue damage state of CFRP structures by measuring surface roughness parameters. Surface roughness and stiffness degradation have been measured in CFRP coupons cycled with constant amplitude loads, and a Pearson's correlation of 0.79 was obtained between both variables. Results suggest that changes on the surface roughness measured in strategic zones of components made of the evaluated CFRP, could be indicative of the level of damage due to fatigue loads. This methodology could be useful for other FRP due to similarities in the fatigue damage process.
\end{abstract}

\section{Introduction}

The growing use of composite materials in aircraft structures, mainly Carbon Fiber Reinforced Polymer (CFRP), makes essential the knowledge of the fatigue damage state, when these structures are subjected to cycling loads of any nature. Examples of these cycling loads include the spectrum loads for wings or constant amplitude loads for fuselages (due to internal pressurization) [1]. A better knowledge of the damage state due to fatigue is useful to optimize the maintenance procedures (repair or replacement) of structural components, keeping the integrity of the structure components in safe conditions.

Conventional techniques to determine the fatigue state of an aircraft structure are based mostly on measurement of structural loads throughout the service life by electric strain gauge sensors, which present some disadvantages. One is that these sensors are affected by extreme environmental conditions such as fatigue loads, electromagnetic fields, etc., in such a way that these sensors require an exhaustive maintenance program. A second disadvantage is that the stiffness degradation of the composite materials due to the accumulated damage on the structure could lead to a non-realistic stress-strain relation on the strain gauge sensors. A third disadvantage is that the accumulated fatigue damage

\footnotetext{
* Correspondence to: Instituto Nacional de Técnica Aeroespacial, INTA, Carretera de Ajalvir, km 4, 28850, Torrejón de Ardoz, Madrid, Spain. Tel.: +34662548450.

E-mail addresses: zuluagarp@inta.es, pzuluag1@gmail.com (P. Zuluaga-Ramírez).
}

determined by load history, is conventionally calculated from linear models initially developed for homogeneous material, where Palmgren-Miner rule is the most extensively used method [2]. Experimental studies show inaccurate and non-conservative predictions when Palmgren-Miner rule is applied to composite materials under spectrum loads [3-5].

There are numerous models developed for fatigue damage accumulation of composite materials, including strength and stiffness degradation $[3,4]$, but due to the complexity of the damage mechanisms on composite materials, models developed are only applicable for specific conditions of loads and materials, and are based on fitting experimental values. The most relevant property to quantify the fatigue damage state of a structure (fatigue damage metric), is the strength degradation of the material due to the accumulated damage (commonly known as residual strength). This relevancy is because there exists a direct relation that the failure is produced when the residual strength becomes equal or lower than the maximum stress applied during cycling $\left(S_{\max }\right)$. Models have been developed to predict the fatigue life of Fiber Reinforced Polymers (FRP) based on strength degradation and several are summarized in the review study developed by Post et al. [3]. In practice, residual strength is a property that cannot be used to determine the fatigue damage state of an inservice structure, because of the impossibility of measuring the strength of a material with non-destructive tests. A second problem is that it is difficult to obtain adequate data to validate models based on strength degradation, because of the combination of two sources of scattering in the experimental data, the first one is the scattering of the initial strength and the second is the scattering of the fatigue 
life. This combination produces a significant scattering on the residual strength measurements [6].

An alternative damage metric to the residual strength method, is to measure the stiffness degradation of the material. Some authors take the stiffness as a fatigue damage metric and establish a relation with the residual strength [7-10]. Stiffness can be measured with non-destructive tests, making easier to follow the evolution of this property throughout the cycles. One disadvantage is that a controlled load should be applied to the structure with a dedicated test setup. A second disadvantage is, as mentioned before, that conventional techniques to measure the stiffness with sensors installed on the component, are affected by fatigue loads and environmental conditions.

Alternative techniques based on phenomenological changes on the composite materials are presented to measure damage due to fatigue loads such as ultrasonic techniques, acoustic emissions [11-13], infrared imaging [14], electrical resistance [15]. Others include non-contact techniques such as thermography, digital image correlation $[16,17]$ and X-ray Tomography [18].

The present work is focused on the evaluation of a new technique to assess the fatigue state of CFRP structures, by means of the evaluation of surface topography variations due to fatigue damage with non-contact measurements. Techniques for evaluating the fatigue state via surface assessment have been developed for structural metals and the conclusion is that the metals undergo a surface transition related to metallurgical effects of their crystal structure [19-22]. A previous approach to evaluate the evolution of surface parameters on CFRP due to fatigue loads was done by the authors [23], where visual inspections show that the change in surface roughness in CFRP materials is a result of micro- or macrosurface cracking of the matrix material and changes of the surface shape due to internal delaminations. In this first study a relation between the evolution of roughness parameters and fatigue cycles at constant amplitude load of $60 \%$ of the ultimate tensile strength $\left(S_{u t}\right)$ has been evaluated [22]. The present study is focused on the evaluation of the evolution of roughness parameters due to the fatigue cycles at different levels of loads and the comparison with a conventional damage metrics such as stiffness degradation.

Roughness parameters have been selected to characterize the topography of the surface, because it is a property that can be measured precisely in the laboratory with nanometer resolution optical perfilometers, such as confocal microscopes, and could be measured in the field by optical techniques such as speckle or portable optical perfilometers [24-27].

\section{Methodology}

\subsection{Materials}

The material selected for the present study is a CFRP type MTM-45-1/IM7 from Advanced Composite Group, ACG, which is a relatively new composite material used for aeronautic structures. Two panels of $2 \mathrm{~mm}$ of thickness in a quasi-isotropic stacking sequence of $\left((45,90,-45,0)_{5}\right)_{2}$, have been manufactured. The panels have been cured in an autoclave at 6 bars and $130^{\circ} \mathrm{C}$ and their quality were verified by standard $\mathrm{C}$-scan ultrasonic test. The ultimate tensile strength $\left(S_{u t}\right)$ of the material is $938 \mathrm{MPa}$ and was statistically estimated in a previous study [23].

For the present study, 13 coupons with dog bone geometry shown in Fig. 1 were extracted from the panels. GFRP tabs were bounded at both ends with film adhesive MTA240 from ACG, in order to obtain a better load introduction and to protect the coupon against the gripping forces of the test machine. The notched geometry of the coupon tests with a gage zone of $10 \times 10 \times 2 \mathrm{~mm}^{3}$ was designed and verified by finite element analysis, in order to guarantee the highest level of strains uniformly distributed at the inspection zone and also to avoid failure due to stress concentration at the interface with the test machine. Finite element results in Fig. 2 are drawn in a normalized scale and show a uniform distribution of strains $(\varepsilon)$ in the direction of the load with variations around $5 \%$ in the gage zone when a tensile load is applied to the coupon.

A test machine (MTS 810 from MTS systems) operated at room temperature under load controlled conditions, was used for the fatigue tests and to extract the stiffness information from the coupons. The surface topography was obtained by a confocal microscope (PL $\mu$ Confocal Imaging Profiler, from Sensofar) with an objective zoom of $50 \times$ and a resolution of $5 \mathrm{~nm}$.

\subsection{Fatigue tests and stiffness measurements}

Cycling loads were applied to 13 specimens under constant amplitude load with a stress ratio of $R=0.1$ (tension-tension load). Different levels of maximum stress $\left(S_{\max }\right.$ ) between $47 \%$ and $66 \%$ of the $S_{u t}$ were applied to obtain the stress life curve $(\mathrm{S}-\mathrm{N})$ shown in Fig. 3. A cycle frequency of $5 \mathrm{~Hz}$ has been selected to prevent overheating. The fatigue tests have been interrupted periodically before failure in order to perform the measurement of the surface parameters.

During the fatigue cycles, the stiffness of the coupons has been measured by the displacement of the test machine and the applied load according to Hooke's law. The machine data was treated in order to obtain the stiffness variations in terms of percentage of the initial stiffness $(E n / E o)$, where Eo is the initial stiffness and $E n$ is the stiffness at cycle $n$.

\subsection{Surface parameters measurements}

Surface topography inspection has been done on both faces of the specimen in an area of $1.55 \times 1.49 \mathrm{~mm}^{2}$ near to the center of the face as shown in the Fig. 1. Due to the stacking sequence, the layer inspected is at $45^{\circ}$ respect to the load direction. The size has been chosen to assess an area with a dimension similar to the area evaluated by laser speckle techniques, in order to have similar statistic and scale. An arbitrary position near to the center of the face has been selected because there is a uniform damage distribution expected along the gage zone of the specimen. The confocal microscope with an objective of $50 \times$ zoom has been used to obtain the topography of the evaluated area, measuring

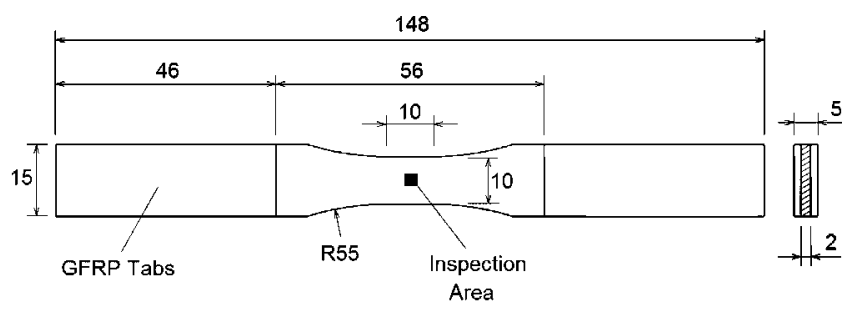

Fig. 1. Coupon geometry, surface inspection area.

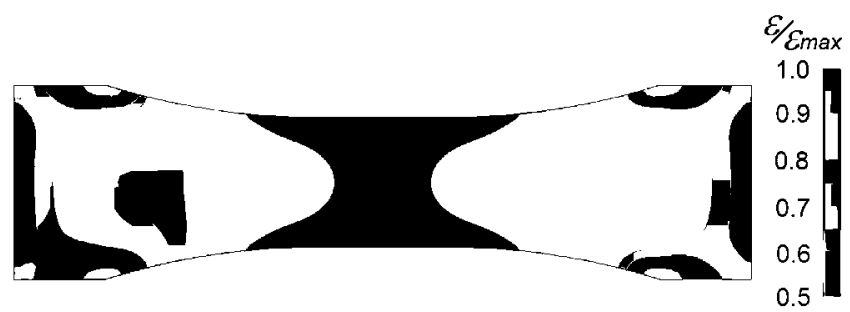

Fig. 2. Normalized strain distribution in the direction of the tensile load by finite element analysis. Variations lower than $5 \%$ of the maximum strain in the gage zone. 
$4675 \times 4478$ pixel. (Taking into account the zoom, each pixel size has an area of $0.33 \times 0.33 \mu \mathrm{m}^{2}$.)

$R a=\frac{1}{P} \sum_{i=1}^{P}\left|z_{i}\right|$

$R q=\sigma=\sqrt{\frac{1}{P} \sum_{i=1}^{P} z_{i}^{2}}$

$R s k=\frac{1}{\sigma^{3} P} \sum_{i=1}^{P} z_{i}^{3}$

$R k u=\frac{1}{\sigma^{4} P} \sum_{i=1}^{P} z_{i}^{4}$

Four parameters were determined from the topography. Those parameters are commonly used to characterize surface roughness based on height magnitudes respect to the mean plane of the surface $[24,25]$. (1) The arithmetic average of absolute values, $R a$. (2) The root mean square (rms or standard deviation), $R q$ or $\sigma$. (3) The third moment, also known as skewness, represents the symmetry of the statistical distribution function, Rsk. (4) The fourth moment, also known as kurtosis, represents the peakedness of the statistical distribution, $R k u$. These parameters are determined with Eqs. (1)-(4) [25], where $z_{i}$ is the value of the height of the measured point $i$ respect to the mean plane of the surface, and $P$ is the total number of measured points (pixels) per surface. (See Fig. 4 for a bi-dimensional scheme.)

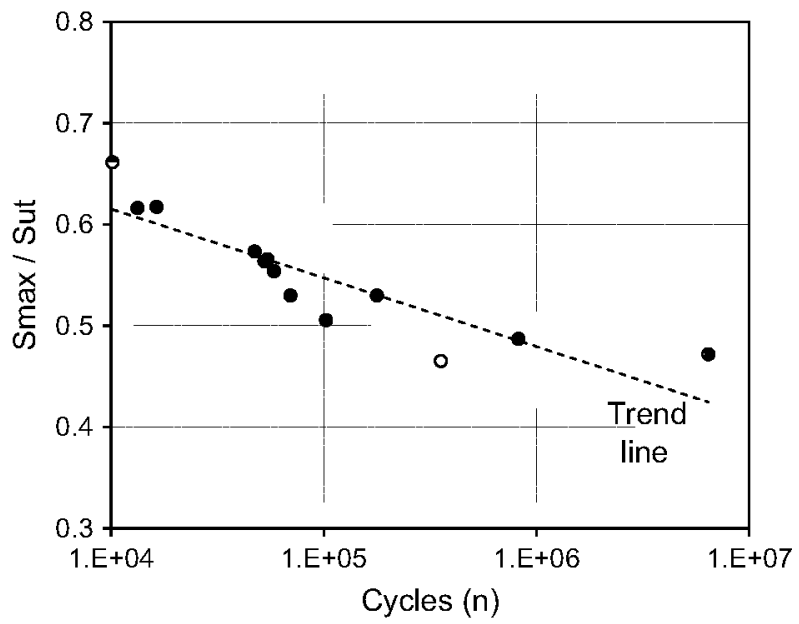

Fig. 3. Stress-life curve (S-N) for $R=0.1$ and average $S_{u t}=938 \mathrm{MPa}$.

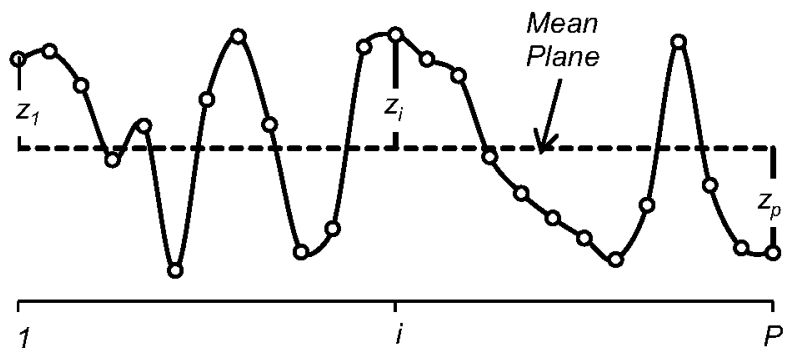

Fig. 4. Bi-dimensional scheme to represent the data measured by the confocal microscope, where " $z_{i}$ " is the value of the height of the measured point " $i$ " respect to the mean plane of the surface, and " $P$ " is the total number of measured points.

\section{Results}

\subsection{Stiffness degradation through cycles}

Fig. 5 shows the variation of the stiffness due to fatigue loads of some representative coupons. The graph A of Fig. 5, shows the evolution of the stiffness variation versus the consumed lifetime $(n / N)$, where $n$ is the applied number of cycles, and $N$ is the number of cycles to failure. The graph B of Fig. 5 shows the stiffness variation versus the cycles applied to each coupon. The stiffness degradation shows a direct relation with the structural damage. One of the observed relation is that the higher the applied load, the faster the degradation of the stiffness; this relation is observed in the graph B of Fig. 5, where higher loads produce curves with higher slopes of stiffness degradation. A second relation is that to fail at lower loads, a higher damage of the structure and in consequence a higher stiffness degradation is required; this relation is observed in the graph A of Fig. 5, where the final value of the stiffness degradation is higher for lower loads.

The coupon $\mathrm{C} 04$ which fails at $N=6.42 \mathrm{E} 6$ cycles (longer life than expected), presents almost the same stiffness degradation compared to the other coupon tested at similar load level (C03) during the first $350 \mathrm{E} 3$ cycles. From this number of cycles the ratio of stiffness degradation of coupon C04 becomes appreciably lower until failure in such a way that the curve on the graph A of Fig. 5 presents two slopes. Visual inspections show large delaminations and high density of matrix cracks from $n=350 \mathrm{E} 3$ cycles until failure, which suggest a change of the damage mechanism that modify the ratio of stiffness degradation, where the load begins to be supported only by the fibers oriented at $0^{\circ}$ (load direction) because of the deterioration of the polymeric matrix.

The stiffness variation and its relation with structural damage, is far away from being in agreement with linear damage accumulation rules. The graph $A$ of the Fig. 5 , shows that at the same level of stiffness degradation, the percentage of life consumed is different depending on the level of load, while the linear damage accumulation rule requires that all the curves for the different coupons tested at different loads would have coincident slopes. As an example, at $90 \%$ of the initial stiffness, the percentage of consumed life for the $\mathrm{CO} 3$ tested at $0.47 S_{u t}$ is near to $20 \%$ versus $80 \%$ for the coupon C14 tested at $0.62 S_{u t}$.

\subsection{Roughness evolution through cycles}

Measurements on the evaluated specimens show variations on the roughness parameters due to accumulated damage. Fig. 6 shows for two specimens, the surface topography evolution of one of the faces due to fatigue loads, with all the measurements presented in the same range of $\pm 4 \mu \mathrm{m}$. Both coupons have been loaded at different levels of $S_{\max }$, the coupon $\mathrm{C} 03$ at $0.47 S_{u t}$, and C14 at $0.62 S_{u t}$. These coupons were selected because they are representative of the phenomena studied in the present work, where the shape of the surface changes due to damage accumulation. The first blocks of cycles produce micro-cracks and local delaminations of the matrix in the surface layers that increase the roughness magnitude of the surfaces (in the present study, the surface layers are orientated at $45^{\circ}$ ). With increasing cycles, the cracks and local delaminations become more profound, and the parameters which measure the magnitude of the roughness of the surface ( $R a$ and $R q$ ) are incremented.

Other observed variations are deviations of the shape of the statistical distribution of the topography, which are measured by skewness and kurtosis parameters. Fig. 7 shows histograms of the heights of the measured points for one of the faces of the specimens C03 and C14, constructed with an binwidth of $0.05 \mu \mathrm{m}$. Regardless 

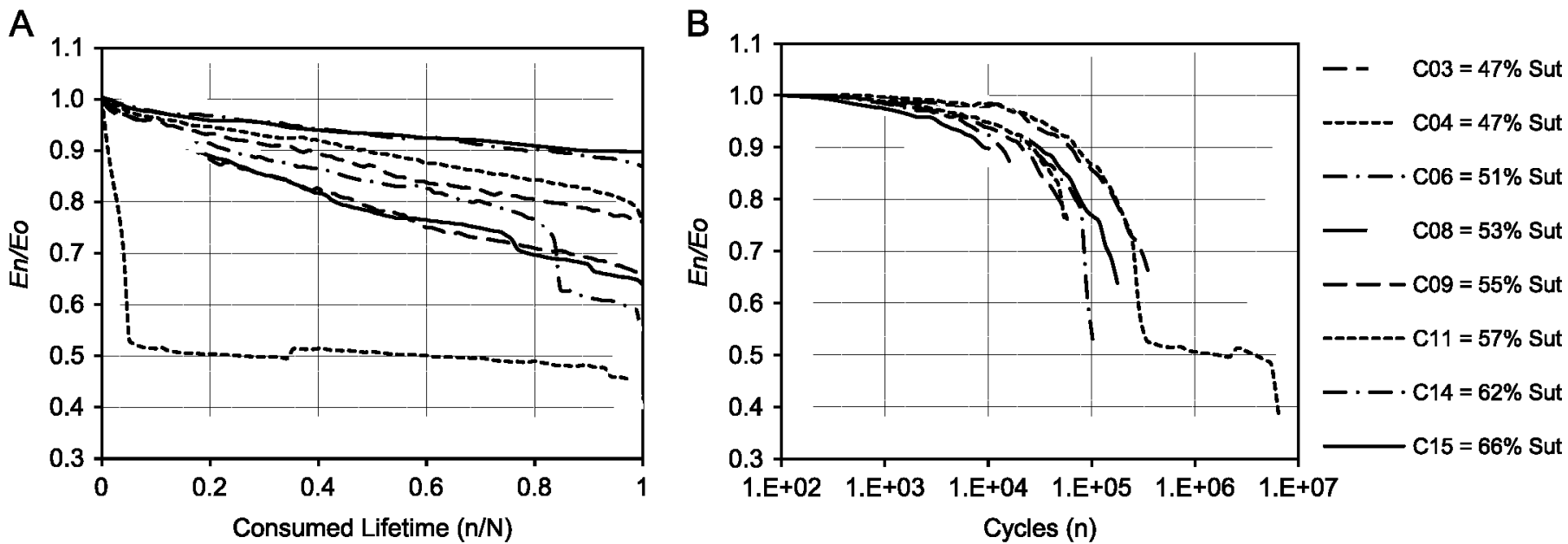

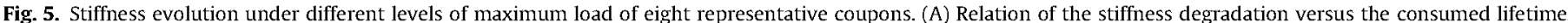
(B) Relation of the stiffness degradation versus the number of cycles in a semi-logarithmic graph.

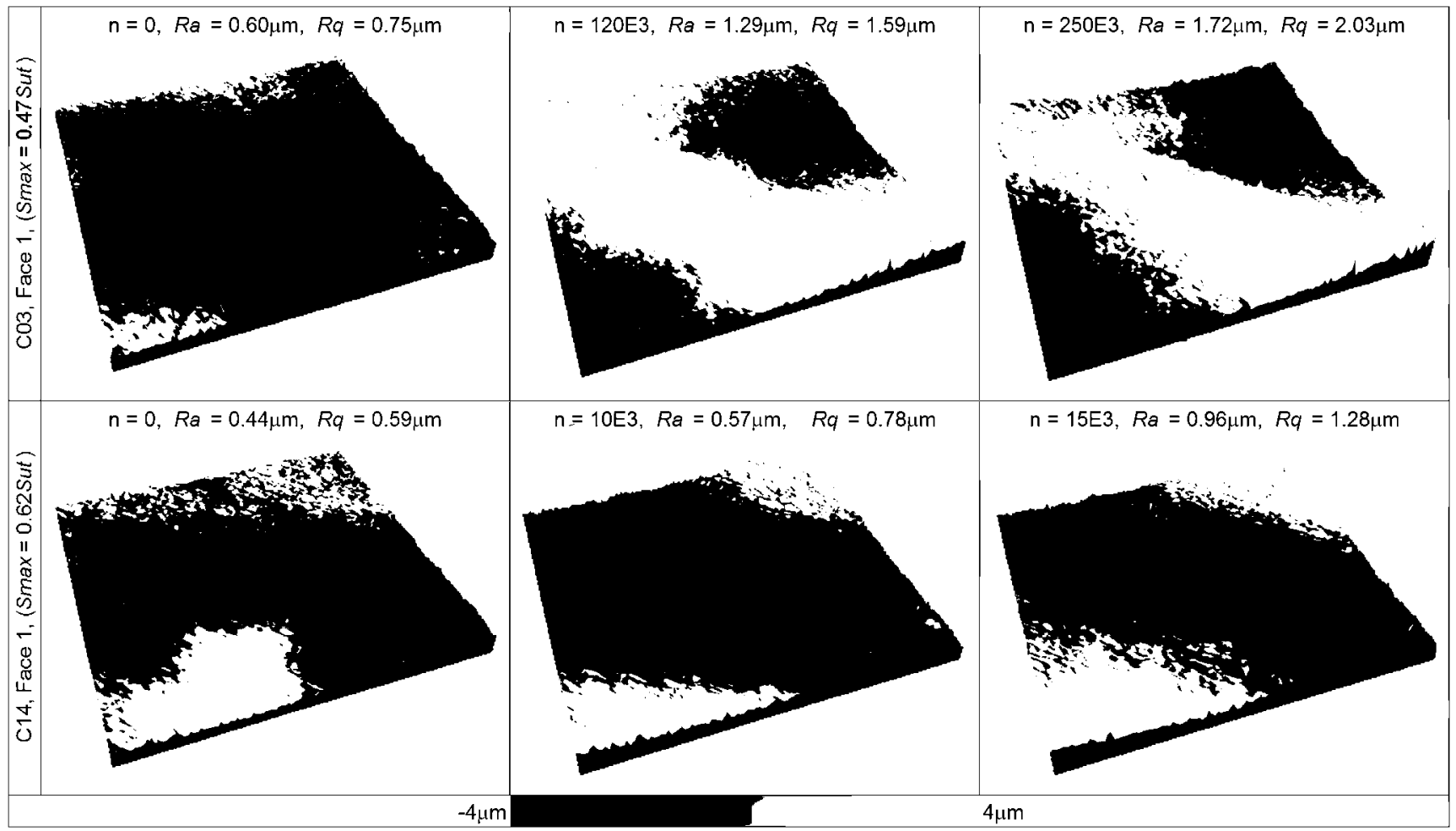

Fig. 6. Surface shape evolution due to fatigue loads for face 1 of coupon $\mathrm{C03}$ loaded at $S_{\max }=0.47 S_{u t}$ and for face 1 of coupon $C 14$ loaded at $S_{\text {max }}=0.62 S_{t i t}$.

A

C03, Face 1, Smax $=0.47$ Sut

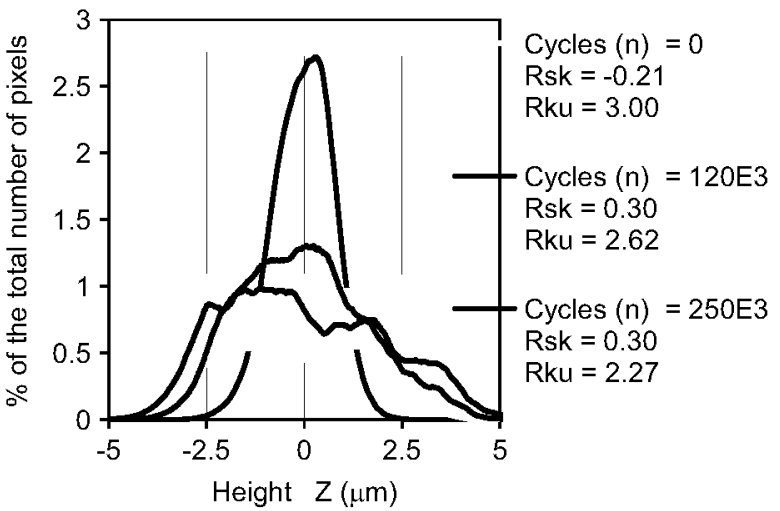

B

C14, Face 1, Smax $=0.62 S u t$

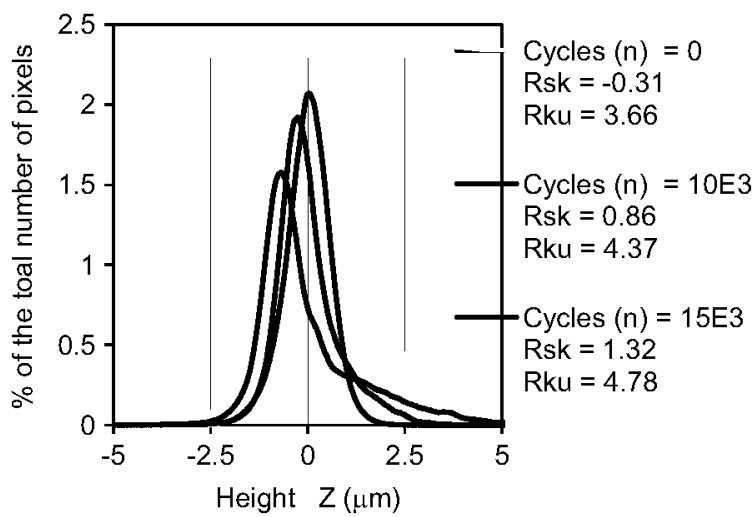

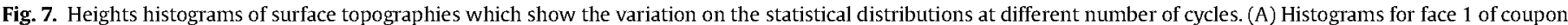
C03 loaded at $S_{\text {max }}=0.47 S_{\text {ut }}$ and (B) histograms for face 1 of coupon C14 loaded at $S_{\text {max }}=0.62 S_{\text {tit }}$. 
of the amount of the standard deviation $(R q)$, it is clear that at the beginning of the life, the topography of the surface is almost Gaussian. Due to the damage accumulation, the distribution becomes asymmetrical and the peakedness changes from the initial quasi-Gaussian shape.

Graphs A-D of Fig. 8, show the variation of the four roughness parameters through the cycles on the evaluated coupons. Parameters measured on each face of the coupon have been averaged to obtain only one value per coupon. $R a$ and $R q$ on graphs $\mathrm{A}$ and $\mathrm{B}$, present a similar behavior because both parameters are used to quantify the magnitude of the roughness. As a general rule, the magnitude of the roughness increases through the cycles due to the accumulation of damage on the material. Rsk value on graph $C$ of Fig. 8 shows as a general rule that the statistical distribution of the surface starts being almost Gaussian or with small negative values of Skewness (with the asymmetry tending to the right), and due to the cycles, the
A

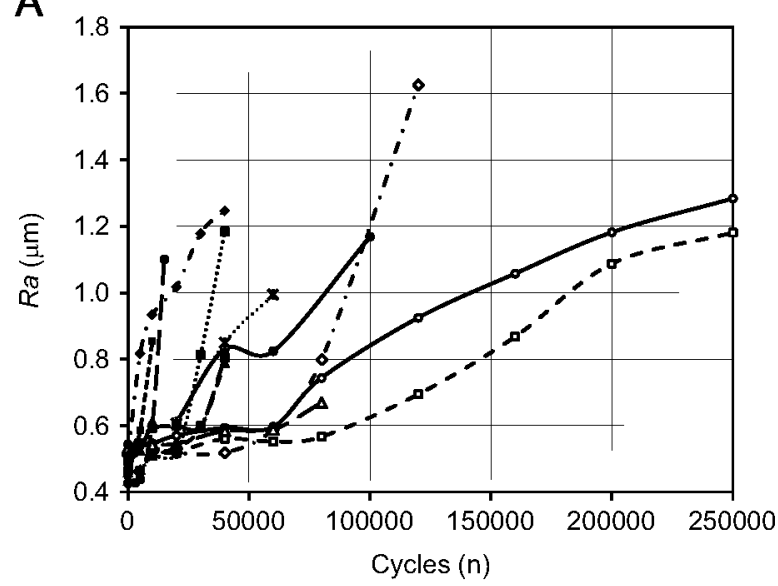

C

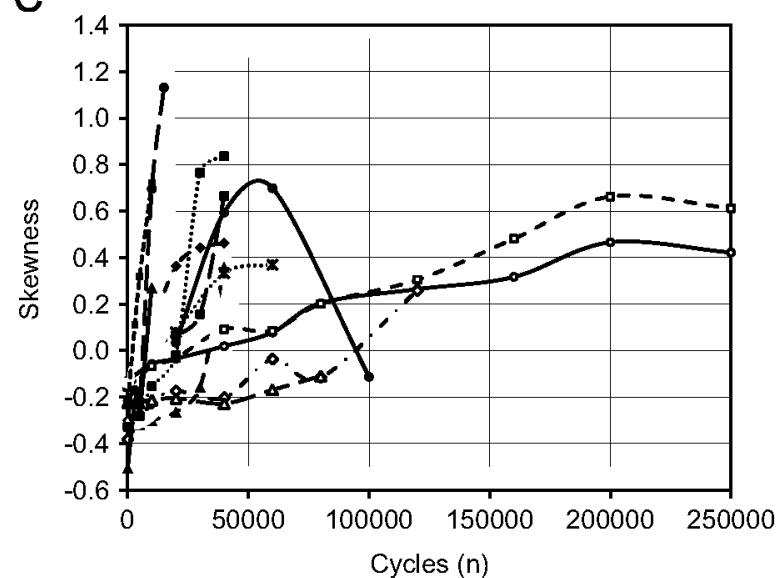

B

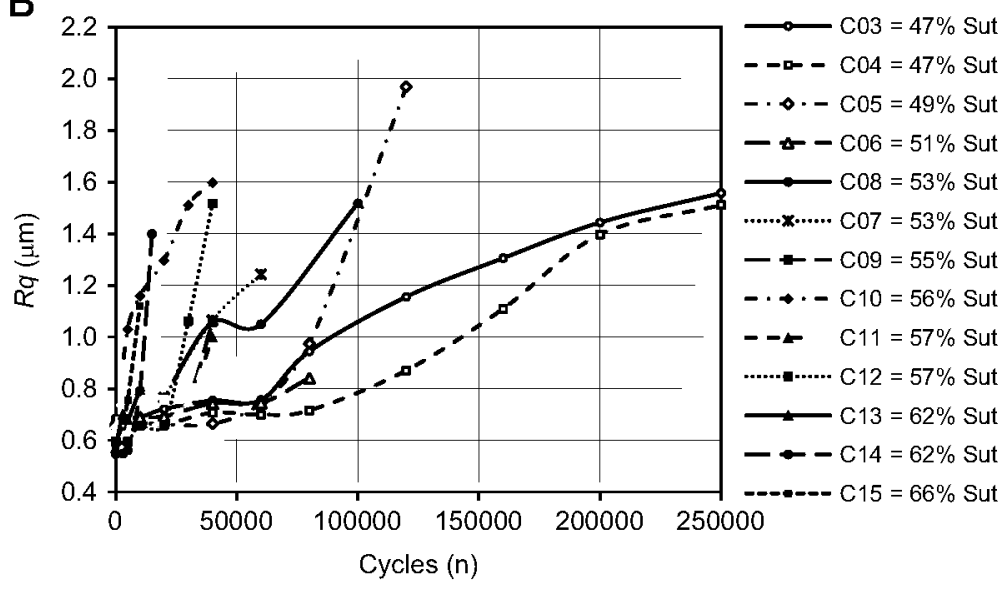

D

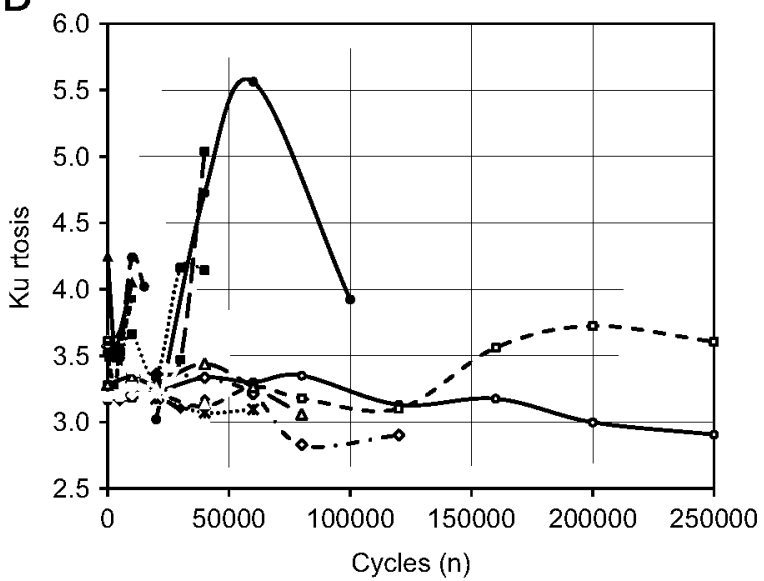

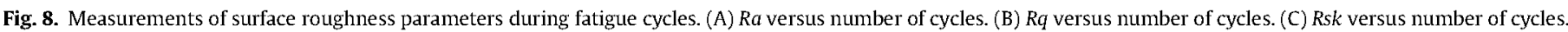
(D) $R k u$ versus number of cycles.

A

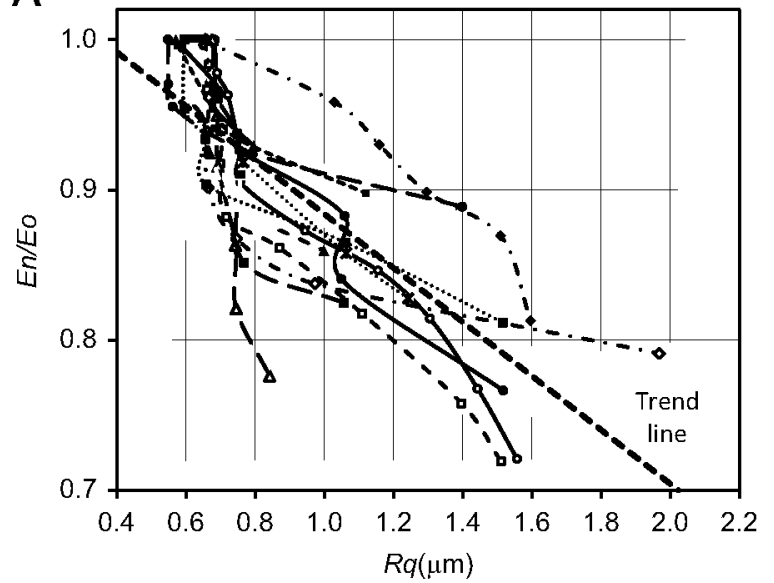

B

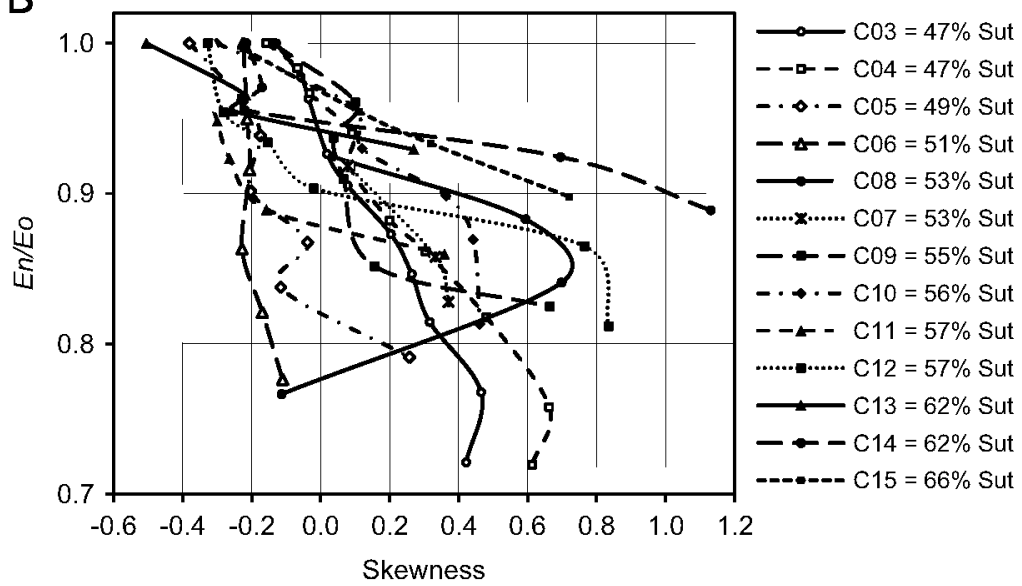

Fig. 9. Relation between surface roughness parameters and the stiffness degradation. (A) $R q$ versus residual stiffness with trend line. (B) Rsk versus residual stiffness. 
asymmetry of the distribution produces positive values of skewness (whit the asymmetry tending to the left). Two coupons present different behavior; one is the CO6 where the Rsk value remains almost the same during the cycles, and the second is the C08 where the last measure of Rsk becomes negative, we can conjecture that this negative value is due to a large delamination present on one of the surface layers. Rku on graph D of Fig. 8 shows changes on the peakedness of the statistical distribution, but not a tendency of increase or decrease.

Two coupons $\mathrm{CO} 3$ and $\mathrm{CO} 4$ were measured only until $n=250 \mathrm{E} 3$, even though failure actually occurred many cycles after this $(N=821 \mathrm{E} 3$ and 6.42E6 respectively). Measurements ceased after $n=250 \mathrm{E} 3$, because the coupons were highly deteriorated due to large delaminations of the surface layers which produced a decoupling with respect to the rest of the laminate in such a way that the load did not flow through the measured layers and the inspection by surfaces parameters was not valid. For the present study we consider as a failure value the total fracture of the coupon, although depending on the inservice applications, a structure could be considered as failed when it reaches a certain level of stiffness degradation, or a level of delaminations, among others.

\subsection{Relation between roughness evolution and stiffness degradation}

Graphs A and B of Fig. 9 shows the relation between the structural damage measured by the roughness parameters, $R q$ and $R s k$, and the damage measured by the stiffness degradation. The parameter $R a$ is omitted because the results of $R q$ shows similar behavior, and the parameter Rku is omitted because does not show conclusive results.

The results on the graph A of Fig. 9, show the agreement between the two damage metrics, in which the magnitude of the roughness tends to increase with the degradation of the coupon stiffness. The Pearson correlation coefficient between both damage metrics has been determined taking into account all the curves, obtaining a value of -0.79 , which confirms that there is a direct correlation between the roughness parameters and the stiffness degradation, and in consequence a direct relation with the damage due to fatigue loads.

The results on graph B of Fig. 9 do not show new conclusions apart of the aforementioned where the value of skewness tends to be positive due to the structural damage.

\section{Conclusions}

There is a growing demand for evaluating the fatigue state of in-service structures or components made of composite materials. The current techniques to evaluate fatigue damage based on nondestructive tests are still in development. This study presents a non-destructive and non-contact technique which determines if a structure or component made of CFRP presents damage due to fatigue based on the assessment of the surface layer.

Experimental results on CFRP coupons show that fatigue loads produce significant changes on the surface topography, where increments on the magnitude of the surface roughness ( $R a$ and $R q$ ) are related to the level of fatigue damage. Roughness measurements were compared to a conventional damage metric such as stiffness degradation instead of linear damage index $(n / N)$, because stiffness degradation is independent on the level of load applied. A linear relation between $R q$ and the stiffness degradation show a Pearson correlation factor of magnitude 0.79.

Fatigue damage also produces changes on statistics of the surface, which starts with a quasi-Gaussian distributions or with negative values of $R s k$, and values of skewness becomes positive due to the fatigue damage. The peakedness of the statistical distribution measured by $R k u$ changes due to the fatigue loads without a clear tendency.

Results suggest that the changes on the surface topography measured in strategic zones of components and structures made of the evaluated CFRP could be indicative of the level of internal damage due to fatigue loads. This methodology could be potentially useful for other FRP structures due to the similarities in the fatigue damage process. These surface inspections could be done on field with non-contact optical techniques such as speckle and optical perfilometers.

\section{Acknowledgments}

The authors would like to thank the INTA Metallic Test Laboratory for their support during the test campaign.

\section{References}

[1] Baker A, Dutton S, Kelly D. Composite materials for aircraft structures. second ed. AlAA Education Series. American Institute of Aeronautics and Astronautics; http://dx.doi.org/10.2514/4.861680.

[2] Boller C, Staszewski WJ. Aircraft structural health and usage monitoring. In: Staszewski WJ, Boller C, Tomlinson GR, editors. Health monitoring of aerospace structures: smart sensor technologies and signal processing. John Wiley \& Sons Ltd.; 2004. p. 29-74.

[3] Post NL, Case SW, Lesko IJ. Modeling the variable amplitude fatigue of composite materials: a review and evaluation of the state of the art for spectrum loading. Int J Fatigue 2008;30(12):2064-86. http://dx.doi.org/ 10 1n16/i iifatigue.2008.07.002.

[4] Epaarachchi JA. A study on estimation of damage accumulation of glass fibre reinforce plastic (GFRP) composites under a block loading situation. Compos Struct 2006;75(1-4):88-92. http://dx.d

5] Zuluaga-Ramîrez P, Frövel M, Arconada Á, Belenguer T, Salazar F. Evaluation of the fatigue linear damage accumulation rule for aeronautical CFRP using artificial neural networks. Adv Mater Res 2014;1016:8-13. http://dx.doi.org/ 10 Anolumenerminntifin mothomr.1016.8.

[6] Wahl N, Samborsky D, Mandell J, Cairns D. Spectrum fatigue lifetime and residual strength for fiberglass laminates in tension. In: proceedings of the 20th ASME wind energy symposium. AIAA, Reno, NV; 11-14 January 2001. AIAA-2001-0025. http://dx.doi.org/10.2514/6.2001-25.

[7] Whitworth HA. Modeling stiffness reduction of graphite/epoxy composite laminates. J Compos Mater 1987;21(4):362-72. $\underline{\text { http://dx.doi.org/10.1177/ }}$ 002199838702100405.

[8] Whitworth HA. A stiffness degradation model for composite laminates under fatigue loading. Compos Struct 1997;40(2):95-101. http://dx.doi.org/10.1016/ 0142-6.

[9] Van Paepegem W, Degrieck J. A new coupled approach of residual stiffness and strength for fatigue of fibre-reinforced composites. Int J Fatigue 2002; 24(7):747-62. http://dx.doi.org/10.1016/S0142-1123(01)00194-3.

[10] Van Paepegem W, Degrieck J. Coupled residual stiffness and strength model for fatigue of fibre-reinforced composite materials. Compos Sci Technol 2002;62(5):687-96. http://dx.doi.org/10.1016/S0266-3538(01)00226-3.

[11] Staszewski WJ, Boller C, Grondel S, Biemans C, O'Brien E, Delebarre C, et al. Damage detection using stress and ultrasonic waves. In: Staszewski WJ, Boller C, Tomlinson GR, editors. Health monitoring of aerospace structures: smart sensor technologies and signal processing. John Wiley \& Sons Ltd.; 2004. p. 125-61.

[12] Adden S, Pfleiderer K, Solodov I, Horst P, Busse $G$. Characterization of stiffness degradation caused by fatigue damage in textile composites using circumferential plate acoustic waves. Compos Sci Technol 2008;68(7-8):1616-23. http: //dx.doi.org/10.1016/j.compscitech.2008.02.031.

[13] Dzenis YA. Cycle-based analysis of damage and failure in advanced composites under fatigue: 1. Experimental observation of damage development within loading cycles. Int J Fatigue 2003;25(6):499-510. http://dx.doi.org/10.1016/ SO14L-11 $\angle 3(02) 00170-6$.

[14] Ahsan M, Han X, Islam S, Newaz G. Fatigue damage detection in graphite/ epoxy composites using sonic infrared imaging technique. Compos Sci Technol 2004;64(5):657-66. http://dx.doi.org/10.1016/j.compscitech.2003.07.005.

[15] Wang $X$, Chung DDL. Self-monitoring of fatigue damage and dynamic strain in carbon fiber polymer-matrix composite. Compos Part B: Eng 1998;29(1):63-73. http://dx.doi.org/10.1016/S1359-8 J14-0.

[16] Dattoma V, Giancane S. Evaluation of energy of fatigue damage into GFRC through digital image correlation and thermography. Compos Part B: Eng 2013;47:283-9. http://dx.doi.org/10.1016/..compositesb.2012.10.030.

[17] Giancane S, Panella FW, Nobile R, Dattoma V. Fatigue damage evolution of fiber reinforced composites with digital image correlation analysis. Procedia Eng 2010;2(1):1307-15. http://dx.doi.org/10.1016/j.proeng.2010.03.142. 
[18] Withers PJ, Preuss M. Fatigue and damage in structural materials studied by Xray tomography. Annu Rev Mater Res 2012;42:81-103. http://dx.doi.org 10.1146/annurev-matsci-070511-155111.

[19] Gordienko YG, Zasimchuk EE, Gontareva RG. Unconventional deformation modes and surface roughness evolution in Al single crystals under restricted cyclic tension conditions. J Mater Sci Lett 2002;21(21):1713-6. http://dx.doi. org/1

[20] Schreiber J, Cikalova U. Fatigue damage evaluation by use of "Smart Sensors". In: proceedings of the 6th European workshop on structural Health Monitoring (EWSHM2012). Dresden, Germany; 3-6 July 2012. tu2c3. 〈http:/www. ewshm2012.com/Portals/98/BB/tu2c3.pdf).

[21] Schreiber J, Cikalova U, Hillmann S, Hoffmann J, Meyendorf N. A fatigue life assessment of aircraft alloys using fractal analysis in combination with eddy current testing. In: proceedings of 18 th world conference on nondestructive testing (WCNDT/18). Durban, South Africa; 16-20 April 2012. (http://www. ndt.net/article/wcndt2012/papers/240_wendtfinal00240.pdf .

[22] Wang L, Wang Z, Xie W, Song X. Fractal study on collective evolution of short fatigue cracks under complex stress conditions. Int J Fatigue 2012;45(0):1-7. http://dx.doi.org/10.1016/j.ijfatigue.2012.06.019.
[23] Zuluaga P, Frövel M, Restrepo R, Trallero R, Atienza R, Pintado JM, et al Consumed fatigue life assessment of composite material structures by optical surface roughness inspection. Key Eng Mater 2013;569-570:88-95. http://dx. doi.org/10.4028/www.scientific.net/kem.569-570.88.

[24] Bhushan B. Surface roughness analysis and measurement techniques. In: Bhushan B, editor. Modern tribology handbook, two volume set. Mechanics \& materials science. CRC press; 2000. p. $49-120$.

[25] UNE-EN ISO 4287:1999. Geometrical product specifications (GPS). Surface texture: profile method. Terms, definitions and surface texture parameters (ISO 4287:1997 + Technical Corrigendum 1).

[26] Salazar F, Belenguer T, García J, Ramos G. On roughness measurement by angular speckle correlation. Metrol Meas Syst 2012;XIX(2):373-80. http://dx. doi.org/10.2478/v10178-012-0032-9, ISSN (Print): 0860-8229.

[27] Léger D, Mathieu E, Perrin JC. Optical surface roughness determination using speckle correlation technique. Appl Opt 1975;14(4):872-7. http://dx.doi.org 10.1364/AO.14.000872 